\title{
ACUTE MEGAKARYOBLASTIC LEUKEMIA
}

\author{
Ana Murtasyidah, Yulia Nadar Indrasari \\ Department of Clinical Pathology, Faculty of Medicine, Airlangga University Dr. Soetomo Hospital, Surabaya, Indonesia. \\ E-mail:yulia.nadar@fk.unair.ac.id
}

\begin{abstract}
Acute Megakaryoblastic Leukemia (AMKL) is a subtype of acute myeloid leukemia triggered by megakaryocytes. Acute megakaryoblastic leukemia is divided into three groups, AMKL in children with Down syndrome (DS-AMKL), AMKL in children who do not have Down Syndrome (non-DS-AMKL), and AMKL in non-DS adults (AMKL adults). The basis of the diagnosis of AMKL or AML-M7, according to $F A B$, is the presence of megakaryocyte line cells as many as $30 \%$ or more of all cells. Meanwhile, the diagnosis of AMKL, according to the 2016 WHO guidelines, is acute leukemia with blasts, about $\geq 20 \%$, $\geq 50 \%$ of which is megakaryocyte line cells. Megakaryocyte cells can be more clearly seen with electron microscopes that react positively to platelet peroxidase or use marker antibodies to CD41/gpIIb, CD42b/gpIb, CD61/gpIIIa, von Willebrand factors, and linker for T cell activation. Based on the results of this research, there are differences in cytogenetics between the three types of AMKL according to their different pathophysiology. The World Health Organization (WHO) argued that AMKL was categorized into not otherwise specific (NOS) AML criteria. These criteria exclude AML with myelodysplasia (AMLMRC), AML associated with therapy, and AML with recurrent genetic abnormalities, such as AML with $t(1 ; 22)$ ( $113.3 ; q 13.1)$, inv (3) (q21.3q26.2), or $\mathrm{t}(3 ; 3)$ (q21.3; q26.2). DS-AMKL is also classified into myeloid leukemia associated with DS. In conclusion, AMKL in adults is not only considered as a rare subtype of $A M K L$, only $1 \%$ of $A M L$ cases in population-based clinical experiments and data but also has a poor prognosis.
\end{abstract}

Key words: Acute megakaryoblastic leukemia, DS-AMKL, non-DS-AMKL, adult AMKL

\section{INTRODUCTION}

Acute Megakaryoblastic Leukemia (AMKL) is a subtype of Acute Myeloid Leukemia (AML) triggered by megakaryocytes. Acute megakaryoblastic leukemia was first described in 1931 by Von Boros but rarely reported in subsequent years due to a lack of consistent diagnostic criteria. Breton-Gorius et al. used an identification technique with Platelet Peroxidase (PPO) to identify small megakaryocytes, thereby increasing the accuracy of the diagnosis of AMKL. ${ }^{1}$ Acute megakaryoblastic leukemia then was categorized by the French-American-British classification (FAB) as AML-M7 in 1985 by providing clearer diagnosis criteria. ${ }^{2}$ The 2016 World Health Organization (WHO) classified AMKL is not otherwise specific (NOS) sub-category. ${ }^{3}$

Acute megakaryoblastic leukemia has a bimodal age distribution with peaks in children aged 1-3 years and adults aged 50-60 years. AMKL consists of $3-10 \%$ of all $A M L$ cases in children and has a poor prognosis, except in children with Down Syndrome (DS) it will have a good prognosis. Acute megakaryoblastic leukemia is the most common type of AML in children with down syndrome which presence is 500 times more than in other children. ${ }^{1,4}$

\section{MEGAKARIOPOIESIS}

Megakaryocytes are the largest cells in the polyploid of bone marrow, which have many copies of chromosomes. Megakaryocytes in healthy bone marrow tissue are affected by various stromal cell cytokines. Megakaryocyte growth is strongly influenced by thrombopoietin (TPO) and other cytokines, such as interleukin-3 (IL-3), IL-6, and IL-11. Megakaryocyte progenitor comes from a common myeloid progenitor which then undergoes several stages of differentiation and maturation. ${ }^{5,6}$

\section{Differentiation of Megakaryocyte Progenitor}

Megakaryocyte progenitor derived from common myeloid progenitor or colony-forming unit granulocyte-erythrocyte-monocyte-megakaryocyte (CFU-GEMM) under the influence of the transcription gene product, GATA-1, regulated by FOG1 cofactors. GATA-1 is a protein product of the GATA-1 X chromosome gene. FOG1 stands for "friend of GATA-1" product of the ZFPM1 gene (zinc finger protein multitype). ${ }^{1}$ Differentiation of 
megakaryocytes is suppressed by another transcription gene product, MYB. Thus, GATA- 1 and $M Y B$ act contrary to produce the megakaryocytopoiesis balance in one line with differentiation of erythropoiesis in another line. ${ }^{6}$

One common myeloid progenitor will appear in three stages of interconnected megakaryocyte progenitor lineages, also called as Megakaryocyte Colony-Forming Cells (Meg-CFC). Each stage is determined by the characteristics of the culture colony in-vitro (Figure 1). However, in the third stage, LD-CFU-Meg loses its capacity to split but still maintained DNA replication and maturation of the cytoplasm. This unique mitotic form of megakaryocytes is known as endomitosis. ${ }^{6,7}$

\section{Endomitosis}

Endomitosis is a form of mitosis in which telophase and cytokinesis do not occur (splits into two cells). When GATA-1 and FOG1 activities slow down, other transcription factors such as RUNX1 trigger changes in mitosis to endomitosis by suppressing the Rho/ROCK signal path so that the actin cytoskeleton is not formed. As a result of suppressing the Rho/ROCK signaling pathway, a series of actin and myosin (muscle fiber like molecules) become inadequate in the shrinking cytoplasms so that there is no division and also cytokinesis.

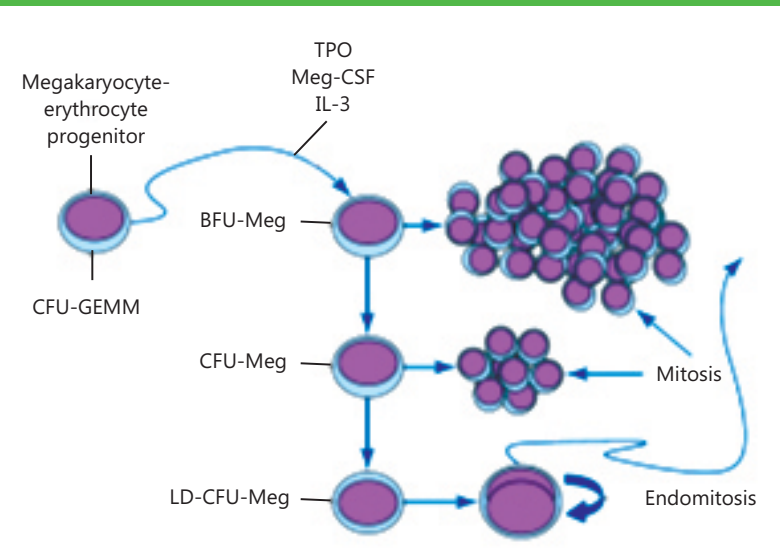

Figure 1. Differentiation of megakaryocyte progenitor ${ }^{6}$

Besides, under the influence of other transcription factors, NF-E2, DNA replication takes place to $8 \mathrm{~N}$, $16 \mathrm{~N}$ and even $32 \mathrm{~N}$ ploidy with multiple chromosomes. Some megakaryocytes replicate four times to reach $128 \mathrm{~N}$. Nevertheless, the level of ploidy is rare and even indicates a hematological disorder. This multiple DNA copy makes the megakaryocytes synthesize many cytoplasms. A megakaryocyte is capable of releasing 2000 to 4000 platelets in a process called as thrombopoiesis. ${ }^{5-7}$

\section{Differentiation of Megacariocyte Terminals}

Furthermore, megakaryoblast (MK-1) begins the development of cytoplasmic ultrastructure, namely

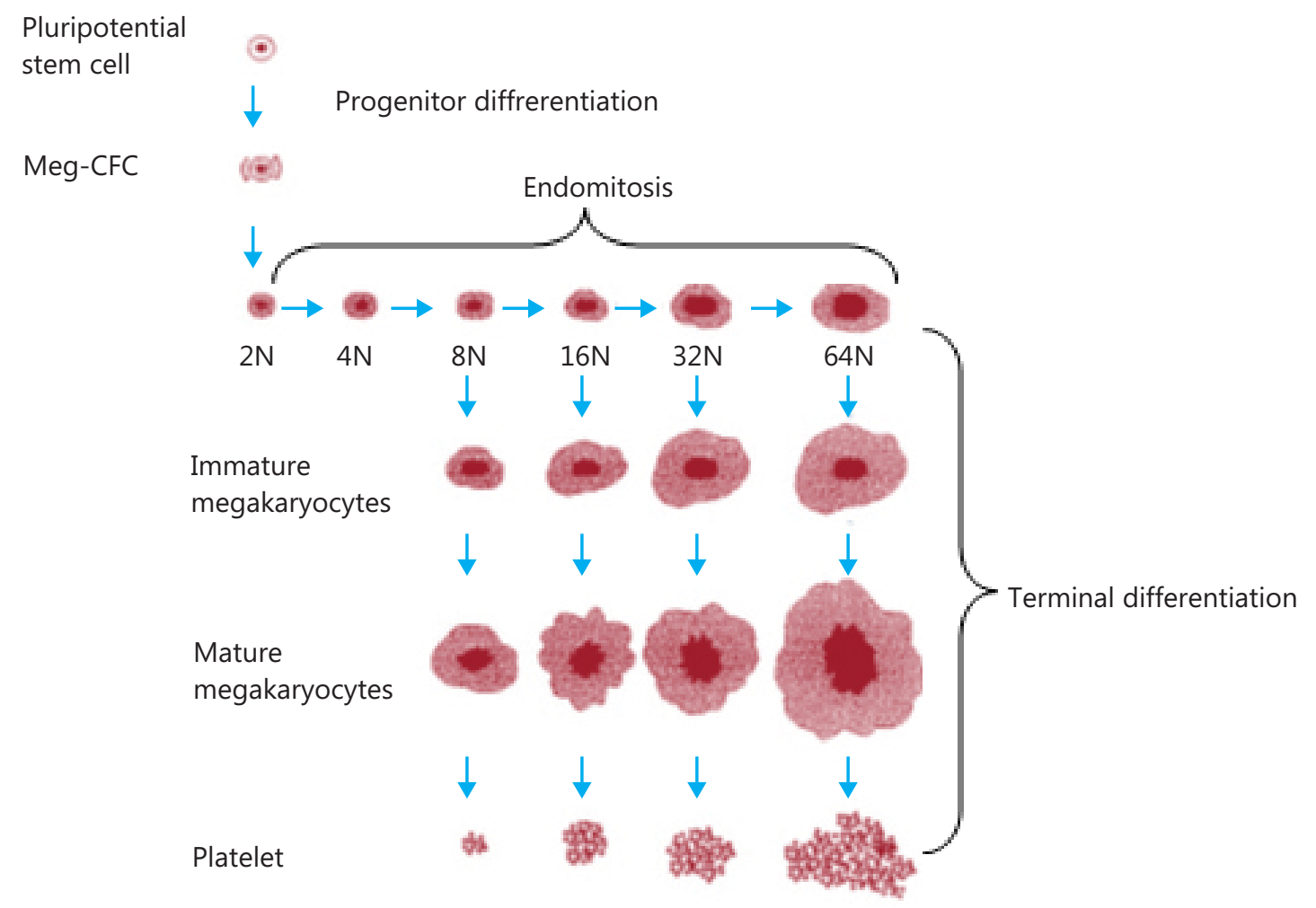

Figure 2. Megakaryopoeisis and trombopoiesis ${ }^{5}$ 
the contents of a procoagulant granules, dense bodies, and Membrane Demarcation System (MDS). The demarcation membrane system shows serial membrane boundary, an invasion of the plasma membrane into the cytoplasm subsequently leading to a subdivision in the cytoplasm. This demarcation membrane system is useful in the preparation of proplatelet membrane material. ${ }^{5,6}$

The MK-II or promegakaryocytes or basophilic megakaryocytes are the developmental stages of endomitosis, from $8 \mathrm{~N}$ ploidy to $64 \mathrm{~N}$ ploidy. ${ }^{5}$ However, other literature states that when core lobularity first becomes clear due to an indentation at the $4 \mathrm{~N}$ replication stage, megakaryocyte cells can be identified as MK-II stages using a light microscope. ${ }^{6,7}$ Fetal and neonatal megakaryocytes that are still produced from central blood even show cytoplasmic maturation on $2 \mathrm{~N} / 4 \mathrm{~N}$ ploidy. Promegakaryocyte phase is identified by an increase in granules that show protein-dense in organelles producing platelets and containing peroxisome. ${ }^{7}$ MK-II composes $25 \%$ of all megakaryocytes. Promegakaryocytes then reach full ploidy level at the end of the MK-II stage. ${ }^{5,6}$

Next, MK-III stage is a stage at which cells looks largest as well as megakaryocytes, are easily identified at $10 x$ objective magnification because the diameter reaches $30-50 \mu \mathrm{m}$. The nucleus is very visible indentation or lobe, but the lobulation level is not proportional to the amount of ploidy. The MK-III stage is characterized by the loss of basophilic cytoplasm alternating with the acidophilic cytoplasm. ${ }^{6}$ Azurophilic granules then appear to be abundant in the cytoplasm and not only seen in centrosomes. Some literature states that there is a development of MK-IV in which the hypnotic nucleus is called as the mature megakaryocyte stage or the megakaryocyte platelet-form, but some other literature consider this stage to be included in the MK-III stage. ${ }^{5}$

MK-I to MK-III stages require 72 hours. However, morphologists rarely make efforts to distinguish MK-I, MK-II, and MK-III stages during routine examination of bone marrow aspiration. Morphological changes in the differentiation of tetra megakaryocyte terminals can be seen in Table $1 .{ }^{6}$

\section{PATHOPHYSIOLOGY AND CLASSIFICATION}

Neonatal megakaryocyte progenitor is in the bone marrow and peripheral blood in which the umbilical cord is the source of stem cells and progenitor cells. Nevertheless, the neonatal megakaryocyte progenitor in adults is only found in the bone marrow. Megakaryocytes from fetal or neonatal progenitors are smaller in size, and the number of ploidy is significantly less than adult megakaryocytes both in-vitro and in-vivo. Megakaryocytes derived from the umbilical cord produce fewer platelets. This indicates that neonatal megakaryocytes are more immature than adult megakaryocytes. Cellular and molecular mechanisms underlie the different pathogenesis of megakaryocyte abnormalities including Transient Myeloproliferative Disorder (TMD) with a picture of megakaryoblasts that only occurs in the fetus and also neonates with DS and GATA-1 mutation. ${ }^{8}$

Acute megakaryoblastic leukemia is derived from a primitive megakaryoblast and consists of megakaryocytes at different ripening levels. Acute megakaryoblastic leukemia leukemogenesis is

Table 1. Morphological changes in megakaryocyte maturation stage ${ }^{6}$

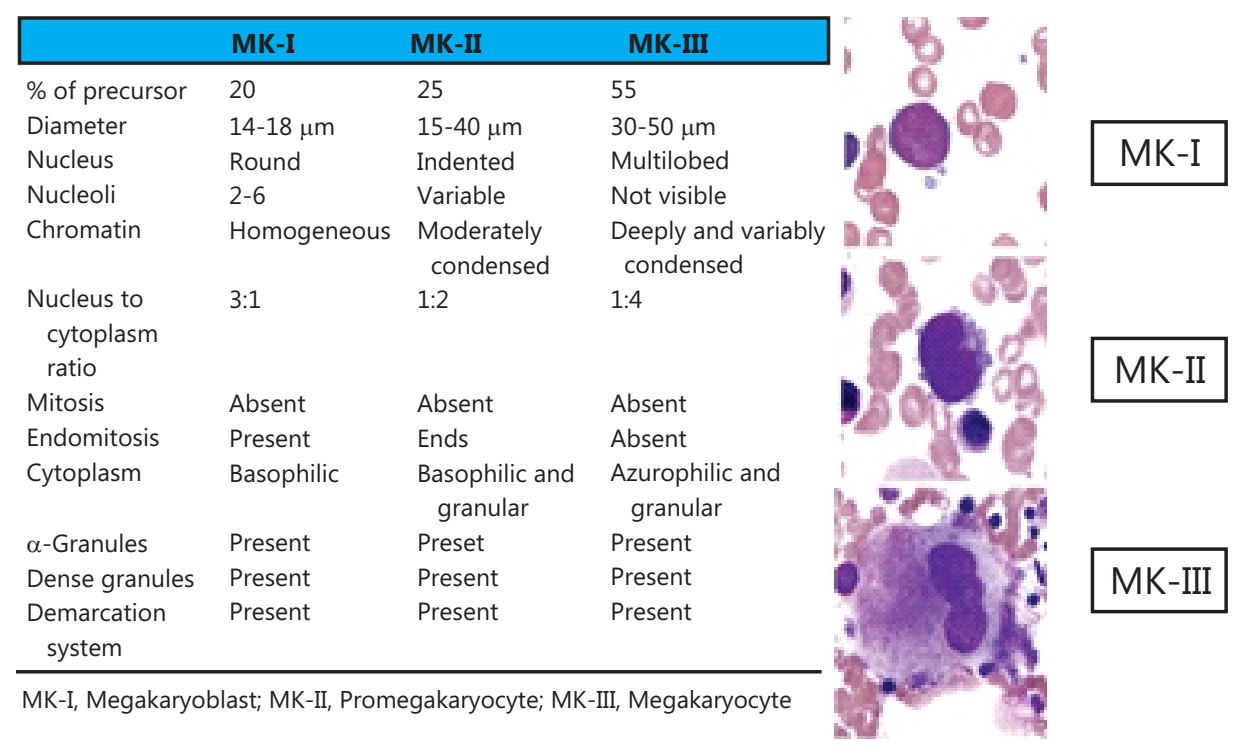


complex and heterogeneous in adults and children. In adults, AMKL can be de novo or secondary as a form of leukemic transformation from previous hematological disorders. Meanwhile, secondary AMKL is often reported from the transformation of Chronic Myelogenous Leukemia ( $C M L)$, Polycythemia Vera (PV), Essential Thrombocytosis (ET), or primary myelofibrosis. ${ }^{1,3}$

Besides, AMKL was divided into three groups based on pathophysiology, age, response to therapy, and prognosis. Those three groups are a group of $A M K L$ children with Down Syndrome (DS-AMKL), a group of AMKL non-DS-AMKL children, and a group of non-DS- AMKL adults. Acute megakaryoblastic leukemia in children without DS is also called as pediatric acute megakaryoblastic leukemia or child AMKL. Acute megakaryoblastic leukemia occurs up to 500 times more in DS children than in children without DS. Non-DS-AMKL and adult-AMKL are rare about $<1 \%$ of all individuals diagnosed as a category of AML-M7.,

\section{Down Syndrome AMKL (DS-AMKL)}

Down syndrome AMKL is very closely related to Transient Myeloproliferative Disorder (TMD), one of the hematological disorders in infants. In this disorder, clonal megakaryoblast populations accumulate in peripheral blood. Transient myeloproliferative disorder patients are mostly known to experience spontaneous remission within three months even without therapy. However, 20\% of TMD cases will develop into Myelodysplasia Syndrome (MDS) and or AMKL.

Transient myeloproliferative disorder, moreover, can be detected from genetic mutation lesions of GATA-1 in fetus in the womb. GATA-1 protein is known to be found typically in erythroid, megacharitic cells, mast cells, and eosinophil lines. GATA-1 is also known to play a role in regulating gene expression controlling not only the maturation of megakaryoblasts into promegacchariocytes, megakaryocytes, and platelets, but also the maturation of erythroblasts into red blood cells. ${ }^{9}$

GATA-1 mutations that underlie the occurrence of TMD, furthermore, involve insertion, deletion, or point mutation in exon 2 leading to transactivation of N-terminal GATA-1 domain, then causing exons shorter. ${ }^{9-12}$ Compared with GATA-1 without mutations, the shorter GATA-1 is less active to stimulate megakaryocyte maturation, but more active to stimulate megakaryoblast proliferation.,10 Thus, the results of this mutation are excessive platelet precursor cell proliferation, decreased circulatory platelets, mild reduction in circulating red blood cells, and TMD development., ${ }^{9,10}$

The frequency of GATA-1 mutations occurs in almost $1 / 3$ of all babies with down syndrome. Meanwhile, TMD occurs in $10 \%$ of down syndrome cases. Besides, 20-30\% of babies with down syndrome and TMD will develop AMKL. Individuals with down syndrome are not only known to have two copies but three copies of chromosome 21 (trisomy 21). Additional copies of the chromosome 21 gene underlie an increased susceptibility to certain inactivation mutations in the GATA gene-1. ${ }^{10,11}$

Besides, the megakaryoblast TMD phenotype functionally does not last long. Blast on TMD is not easy to grow and develop outside the bone marrow. In contrast to $A M K L$, blast in $A M K L$ contains more mutations with the addition of epigenetic lesions and kinase coding genes making the disease more progressive. Additional genetic lesions can occur later and then cause a leukemia. ${ }^{10,11}$

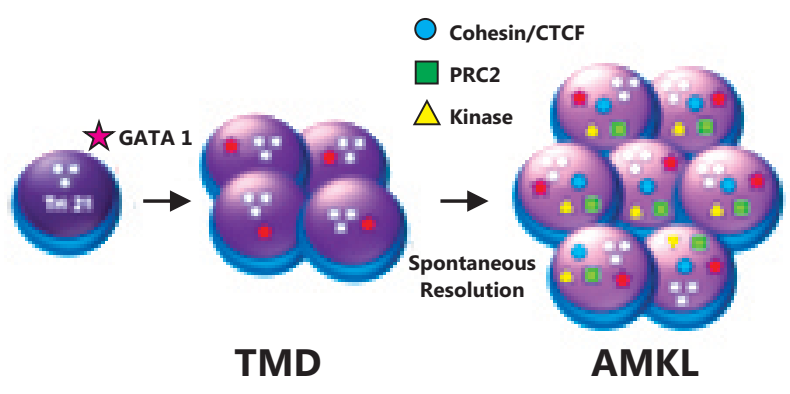

Figure 4. The process of DS-AMKL disease ${ }^{11}$

Down syndrome in the fetus and neonate with a GATA-1 mutation is rarely asymptomatic (silent TMD). The fatal incidence of this disease is about $20 \%$ of the cases, but $80 \%$ of infants with TMD fully recover from the disease within four months. However, DS-AMKL in patients with a history of $10 \%$ TMD, both symptomatic and silent, can be developed within 4 years. Individuals who have experienced this GATA-1 mutation during this period can also experience somatic megakaryoblast mutation affecting cohesin, epigenetic regulators, and signal molecules. This newly acquired mutation arises as a result of the interaction of GATA-1 mutation and excessive chromosome 21 gene copies. Genes that are thought to be involved are megakaryoblastregulatory genes, namely TP53, FLT3, ERG, DYRK1A, CHAF1B, HLCS, RUNX1, and MIR125B2 (which is the MiR125B2CTCF microRNA gene), complex cohesin genes including STAG2, 
RAD21, SMC3, SMC1A, and NIPBL, as well as epigenetic regulatory genes including SUZ12 and PRC2. Meanwhile, gene mutations that affect signal molecules include JAK1, JAK2, JAK3, MPL, KRAS, NRAS, and SH2B3. . $^{1,9,10,11}$

\section{Pediatric AMKL (Non-DS-AMKL)}

The rearrangement of the genes then produces imer chimeric oncogene identified in non-DS AMKL. But, $30-40 \%$ of the cases cannot be identified. De Rooij et al. examining RNA and or 'exome sequencing' of 75 children and 24 adults who experienced AMKL found that fusion of genes occurred in $5.5 \%$ of adult patients and $72.4 \%$ of pediatric patients. ${ }^{12}$ The research also revealed that the gene fusions found in non-DS AMKL were CBFA2T3-GLIS2 (18.6\%), KMT2A (17.4\%), NUP98-KDM5A (11.6\%), and RBM15-MKL1 (10.5\%).

Another previous research on gene fusion involving several HOX rearrangements occurred in $14 \%$ of patients. In the research, AMKL cases that were not identified with fusion were $21 \%$, half of which had GATA1 cutting mutations. Non-DS AMKL whole-exome sequencing shows GATA1, JAK or STAT mutations, cohesins or CTCF genes, and RAS pathway genes. Fusions of RBM15-MKL1 and CBFA2T3-GLIS2 are the most common fusions. RBM15-MKL1 has the best prognosis among other child AMKL abnormalities. ${ }^{7}$ Meanwhile, the prognosis of CBFA2T3-GLIS2 is the worst one. ${ }^{10}$

One of child non-DS-AMKL or AMKL genetic abnormalities is a non-reciprocal translocation between short arms or between $p$ arm at position 13 on chromosome 1 (1p13) and parm at position 13 on chromosome 22 (22p13). This translocation, $\mathrm{t}(1 ; 22)$ (p13; q13), occurs mainly in infants. But, it is also seen in AMKL children aged up to 7 years. This translocation involves RNA-binding motif protein-15 gene (RBM15 gene) often called as OTT ("one twenty-two") on chromosome 1, and megakaryocyte leukemia-1gene (MKL1 gene) often called as Megakaryocytic Acute Leukemia (MAL) on chromosome 22 to make RBM15-MLK1 (OTT-MAL) fusion gene. ${ }^{11}$

The RBM15-MLK1 fusion protein causes deregulation, so it suppresses the MLK1 target gene. But, SRF is activated even though without stimuli. The fusion of genes also interferes with the stimulation of RPBJ target genes. This triggers the notch signal path to be too active. Mice conditioned to experience chimeric oncogenic disorders in the promoter RMB15 then turned out to show hematopoietic disorders as in their fetus and adulthood. Some even developed into AMKL at the age of 18-24 months. However, it should be suspected that these processes must be accompanied by other oncogenic processes to explain the development of non-DS AMKL in humans. $^{11}$

Chromosome 16 inversion, inv (16) (p13.3q24.3) is detected to produce fusion of CBFA2T3 gene and GLIS2 gene. The CBFA2T3 gene belongs to the ETO core receptor group, while GLIS2 belongs to the GL1 transcription factor group. The expression of the CBFA2T3-GLIS2 fusion gene in Drosophila hematopoietic cells and experimental mice induces a Bone Morphogenetic Protein (BMP) signal causing an increase in the self-renewal capacity of progenitor cells. Cells expressing CBFA2T3-GLIS2 remain dependent on growth factors in-vitro. Nevertheless, it is assumed that there are other mutations associated with signal growth factors for the occurrence of leukemia. ${ }^{11}$

\section{Adult AMKL}

Adult $A M K L$ can be derived from the development of other myeloproliferative (MPN) neoplasms, chronic myeloid leukemia, polycythemia vera, essential thrombocytosis, and primary myelofibrosis. ${ }^{1,3}$ In a study on AMKL in adults, $25 \%$ of 49 cases are considered as a secondary to one of those MPNs. ${ }^{13}$ Unfortunately, the mechanism behind this secondary AMKL case is still unknown even though inversions or translocations on chromosome 3 at positions q21 and q26 inv (3) (q21q26) or t (3: 3) (q21q26) are often seen as the secondary cases of AMKL in adult. ${ }^{1,3}$

AMKL in some cases, moreover, is found in adults who also have tumors of mediastinal germ cells. ${ }^{1,3,5}$ These tumors are germ cell malignancies, which are primitive cells that give rise to sperm cells and egg cells. The germ cell mediastinaltumor associated with AMKL in adults is not seminoma and occurs before or together with, but not after the diagnosis of AMKL. The three most common genetic aberrations in bone marrow cells of these individuals (representing 65\% of all cases) are inversions in the $p$ arms of chromosome 12, trisomy 8 , and the extra $X$ chromosome. In some of these cases, genetic deviations in malignant platelet precursor cells are similar to those of mediastinal germ cell tumors. The results of the analysis indicate that both malignancies originate from the same cell clone., ${ }^{3,14}$ Overall, the most common genetic deviations in adult $A M K L$ as described previously are inversion inv (3) (q21q26), $t$ (3: 3) (q21: q26), t (9:22) (q34: q11), and various 
deviations in chromosome 5 or chromosome 7. The last two chromosome deviations are also usually seen in AML associated with changes related to myelodysplasia in which immature blood cells dominate in the bone marrow. ${ }^{1}$ The mechanism of the underlying malignancy behind this genetic aberration, however, still needs further research.

\section{DIAGNOSIS}

The diagnosis of AMKL or AML M7, according to French-American-British criteria (FAB), is established using bone marrow aspiration detecting the presence of megakaryocyte cell lines as much as $>30 \%$ of all cells. Myelofibrosis due to increased reticulin often occurs in AML M7 patients so that adequate sampling of bone marrow is difficult to obtain. Calculation of blast on the bone marrow aspiration, unfortunately, can be incorrect. Calculation of the number of blasts can be conducted if an adequate bone marrow biopsy is obtained. The incidence of bone marrow fibrosis in AMKL cases even can lead to no significant cytological findings so that examination of peripheral blood smear must be performed. The presence of the megakaryocyte line can be identified using immunological techniques in the smear of peripheral or bone marrow blood.

The classification of hematological malignancy, according to WHO (2016), categorizes AMKL into the criteria of not otherwise specific (NOS) AML. AMKL is acute leukemia with $>20 \%$ blast cells, $>50 \%$ of which are the megakaryocyte lines. These criteria exclude $\mathrm{AML}$ with myelodysplasia (AML-MRC), AML-related therapy, and $A M L$ with recurrent genetic abnormalities, such as AML with $\mathrm{t}(1 ; 22)(\mathrm{p} 13.3 ; \mathrm{q} 13.1)$; RBM15-MKL1, inv(3) (q21.3q26.2), or $\mathrm{t}(3 ; 3)(\mathrm{q} 21.3 ; \mathrm{q} 26.2)$ (GATA-2, MECOM). Down syndrome-AMKL is also classified into myeloid leukemia associated with DS. ${ }^{3}$
Patients with AMKL usually experience cytopenia although in some cases thrombocytosis occurs. Dysplasia is often found in all cell lines. Dysplasia can be found in neutrophils, erythroid precursors, platelets, and megakaryocytes, but does not meet the criteria for AML-MRC. The diagnosis of AMKL requires the minimum presence of $20 \%$ blasts, $50 \%$ of which (the minimum one) are derived from the megakaryocyte line.

\section{Morphological Examination of Megakaryoblast Cells}

Morphological examination is generally carried out using Wright staining technique with a light microscope. The diameter of the megakaryoblast cells varies from the size of small lymphocytes to three times its size. Megakaryoblasts are generally in various sizes, from medium to large (12-18 pm) ones, with rounded nuclei and slightly irregular forms, or with indents in forms of fine reticular chromatin and 1-3 nucleoli. In peripheral blood, micromegakaryocytes, megakaryoblast fragments, large platelet dysplasia, and hypogranular neutrophils can be found. Micromegakaryocytes, nevertheless, should not be counted as blasts. ${ }^{3,6}$

The clinical diagnosis of AMKL from bone marrow histology is still difficult to distinguish acute panmyelosis from myelofibrosis (APMF). However, the diagnostic criteria of the $F A B$ classification system and the emergence of flow cytometry can increase diagnostic sensitivity for AMKL. ${ }^{1}$ Megakaryocytes activate fibroblast factors so that they cause secondary extensive fibrosis in the bone marrow. ${ }^{1}$ Extensive fibrosis seen in AMKL causes inadequate bone marrow aspirates (dry tap), thus often causing failure of the cytogenetic analysis and difficulty in determining the exact number of blast cells present in the bone marrow, needed to differentiate AMKL from APMF. ${ }^{1,3,5}$ Although dry tap is a problem, adequate calculation of blast cells is

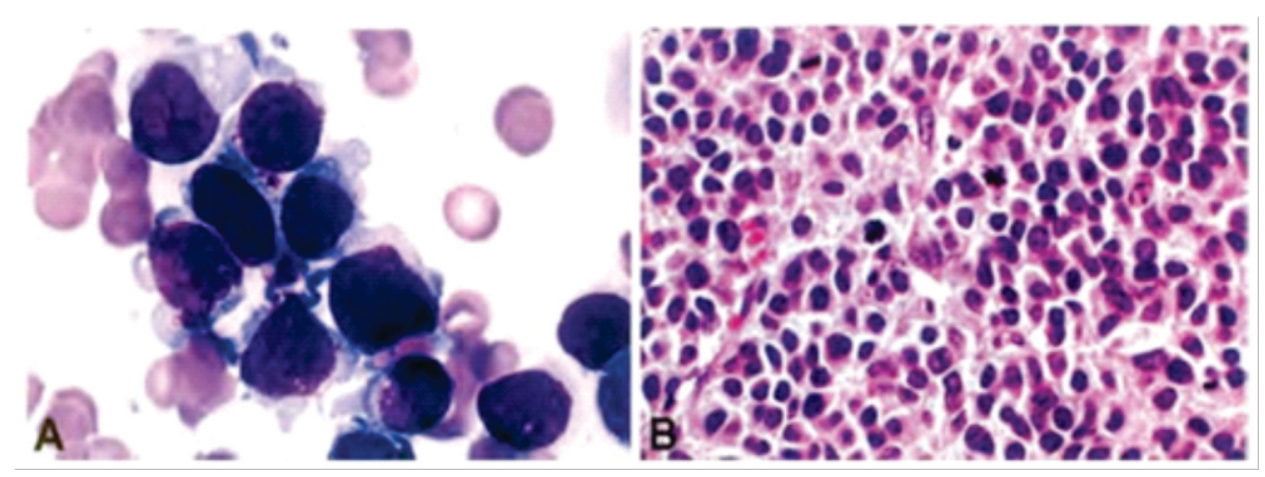

Figure 5. $A M K L$ with Wright staining technique on bone marrow smear $(A)$ and bone marrow biopsy $(B)^{3}$ 
obtained from a bone marrow biopsy using immunohistochemical staining. ${ }^{1,3}$

Cytochemical staining of megakaryoblasts, such as on Sudan Black B, Naphthol AS-D, Chloroacetate Esterase (CAE), and Myeloperoxidase (MPO) consistently gives negative results. The results can be positive in the Periodic Acid-Phosphatase (PAS) reaction against phosphatase acid and"punctata" (non-specific focal reactive esterase). Platelet peroxidase activity is more clearly seen using an electron microscope to identify megakaryoblast cells in AMKL. Platelet peroxidase reactions are almost always positive in AMKL. This examination is either performed on peripheral blood preparations or fresh (not fixed) bone marrow or fixation with tannic acid followed by formaldehyde and glutaraldehyde. , $^{2,3}$

\section{Immunophenotyping}

The morphology of megakaryoblasts or megakaryocytes in AMKL can appear diverse with the presence of dysplasia. It can be observed at laboratories with immunological painting techniques using marker cells. Megakaryoblasts express platelet glycoprotein Cd41 (glycoprotein IIb), CD42b (glycoprotein Ib), and CD61 (glycoprotein IIIa). The expressions of CD41 or CD61 from the cytoplasm are more specific and sensitive than cell surface painting since the possibility of platelet attachment in the blast (satellism) can cause false-positive interpretation of flow cytometry. ${ }^{3,5,6}$

A study shows that expressions of CD41/GPIIb and CD61/GPIIIa may be seen in megakaryocytes which maturity is faster. Meanwhile, CD42b is more positive in some cases even with a small number of blast cells. ${ }^{1}$ Bone marrow specimens using trephine biopsy can be used in making a diagnosis. Megakaryocytes or megakaryoblasts can also be identified with a positive reaction to antibodies of the von Willebrand factors. Platelet glycoprotein (CD61 and CD 42b) and LAT staining (linker for activation of $\mathrm{T}$ cells) are the most specific markers, but the detection of them is very dependent on the fixation and decalcification procedures. ${ }^{3}$

Myeloid markers, CD13 and CD 33, are positively expressed, so are CD2, CD7, and CD56. CD45 and HLA-DR panleukocyte markers. But, they also often indicate negative results, especially in children. CD34 is positive in $60 \%$ of cases. CD36 is a marker of $A M K L$ characteristics, but it is not specific. Blast has a negative result on MPO and is also considered as a marker of granulocytic differentiation. Lymphoid and TdT markers are not expressed, but sometimes lead to aberrance of CD7. ${ }^{1,3}$ AMKL in children with DS has different immunophenotypes precisely in the presence of $\mathrm{CD7}$, antigen expression related to atypical lymphoid, CD11b, and positive CD36 not found in other AMKL subtypes. ${ }^{1}$

\section{Cytogenetic Examination}

Acute megakaryoblastic leukemia karyotypes are more complex when compared to other AML subtypes although they have been separated between child and adult subtypes. Genetic abnormalities found in DS-AMKL associated with trisomy 21 have mutations in GATA1 and other somatic mutations as mentioned earlier. Many AMKL abnormalities in children, according to WHO in 2016, were categorized in the acute myeloid leukemia criteria with current genetic abnormalities, namely $t$ $(1 ; 22)$ (p13.3; q13.1) forming the fusion gene of RBM15-MKL1, inv (16)(p13.3q24).3) then forming the fusion gene of the CBFA2T3-GLIS2 and $t(11 ; 12)$ (p15.5; p13.5) leading to the formation of NUP98-KDM5A fusion fusion. However, there are no typical chromosomal abnormalities associated with adult AMKL. The typical complex karyotypes in myelodysplastic syndrome, inv (3) (q21.3q26.2) and $t$ (3: 3) (q21.3q26.2), are thought to be related to the differentiation of megakaryocytes or megakaryoblasts. $^{3}$

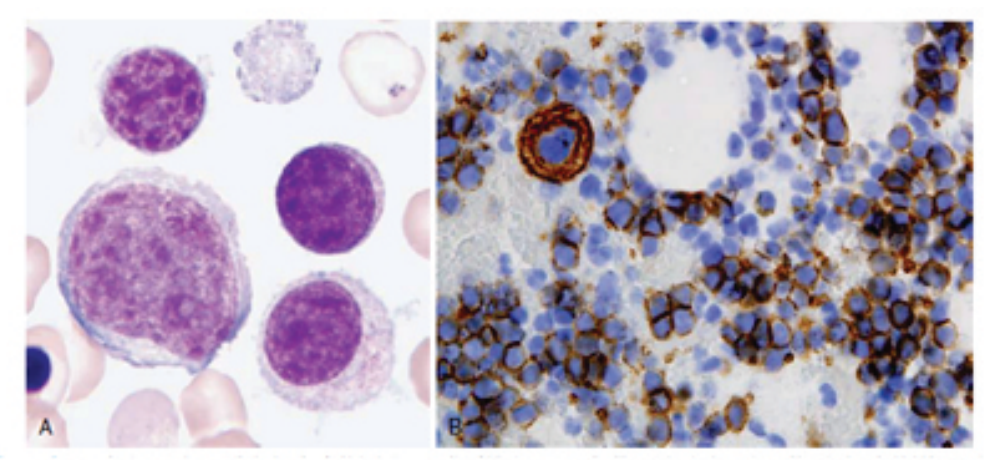

Figure 6. Acute megakaryoblastic leukemia (A) Cytoplasmic blebbingin blast (B) Positive reaction against CD42b. ${ }^{3}$ 


\section{PROGNOSIS}

The prognosis of AMKL in adult patients treated is far below other AMKL types. The average survival time is only 18 to 41 weeks with a 5-year survival rate of only 10-11 percent. As a result, due to the statistical results, a new treatment approach required should be directed at the mechanisms underlying this disease. ${ }^{1}$

\section{REFERENCES}

1. Hahn AW, Li B, Prouet P, Giri S, Pathak R, Martin MG. Acute megakaryocytic leukemia: What have we learned. Blood Reviews, 2016;30(1):49-53.

2. Bennett JM, Catovsky D, Daniel MT, Flandrin G, Galton DA, Gralnick HR, et al. Criteria for the diagnosis of acute leukemia of megakaryocyte lineage (M7). A report of the French-American-British Cooperative Group. Ann Intern Med, 1985;103:460-2.

3. Swerdlow SH, Campo E, Harris NL, Jaffe ES, Pileri SA, Stein $\mathrm{H}$, Thiele J (Eds): WHO classification of tumors of hematopoietic and lymphoid tissues (Revised $4^{\text {th }}$ edition).IARC, Lyon, 2017; 138-139, 162-164.

4. Gruber TA, Downing JR. The biology of pediatric acute megakaryoblastic leukemia. Blood, 2015;126(8: 943-9.

5. Kaushansky K, Lichtman MA. Williams hematology, Ninth Ed., New York, McGraw Hill Education. 2016; 13:1815-27; 1392.

6. Fritsma GA. Platelet production, structure, and function In Rodak's hematology: Clinical principles and applications. Fifth Ed.,St.Louis, Elsevier Inc. 2016; 13: 167-172.

7. Orazi A, Foucar K. Knowles neoplastic hematopathology. Third Ed., Philadelphia, Lippincott Williams \& Wilkins, 2014; 21-23.

8. Liu Z, Sola-Visner. Neonatal and adult megakaryopoiesis. Curr Opin Hematol. 2011; 18:330-337.

9. Seewald L, Taub JW, Maloney KW, McCabe ER. Acute leukemias in children with down syndrome. Molecular Genetics and Metabolism. 2012;107(1-2): 25-30.

10. Bhatnagar N, Nizery L, Tunstall O, Vyas P, Roberts I. Transient abnormal myelopoiesis and AML in Down syndrome: An update. Current Hematologic Malignancy Reports, 2016;11(5): 333-41.

11. Gruber TA, Downing JR. The biology of pediatric acute megakaryoblastic leukemia. Blood, 2015;126(8): 943-9.

12. de Rooij JD, Masetti R, van den Heuvel-Eibrink MM, Cayuela JM, Tarka J, et al. Recurrent abnormalities can be used for risk group stratification in pediatric AMKL: A retrospective intergroup study. Blood, 2016; 127(26): 3424-30.

13. Wang SA, Hasserjian RP. Acute erythroleukemias, acute megakaryoblastic leukemia, and reactive mimics: A guide to a number of perplexing entities. American Journal of Clinical Pathology, 2015;144(1) :44-60.

14. Gassmann W, Loffler H. Acute megakarioblastic leukemia. Leuk Lymphoma, 1995;18(1): 69-73. 